\title{
Erratum to: Patent litigation in Europe
}

\author{
Katrin Cremers ${ }^{1} \cdot$ Max Ernicke ${ }^{2} \cdot$ Fabian Gaessler $^{3}$. \\ Dietmar Harhoff ${ }^{3}$. Christian Helmers ${ }^{4}$. \\ Luke McDonagh ${ }^{5} \cdot$ Paula Schliessler $^{1}$. \\ Nicolas van Zeebroeck ${ }^{6}$
}

Published online: 4 April 2016

(C) Springer Science+Business Media New York 2016

\section{Erratum to: Eur J Law Econ \\ DOI 10.1007/s10657-016-9529-0}

Owing to an unfortunate error during the production process, Table 9 was published with incorrect spanning of table headers. Therefore, it has been corrected with this erratum (Table 9).

The online version of the original article can be found under doi:10.1007/s10657-016-9529-0.

Dietmar Harhoff

dietmar.harhoff@ip.mpg.de

1 ZEW, Mannheim, Germany

2 Universität Düsseldorf, Düsseldorf, Germany

3 Max Planck Institute for Innovation and Competition, Munich, Germany

4 Santa Clara University, Santa Clara, CA, USA

5 City University, London, UK

6 Université Libre de Bruxelles, Brussels, Belgium 
Table 9 Outcomes of German cases with parallel case ${ }^{\mathrm{a}}$ in UK, France and Netherlands (2000-2008)

\begin{tabular}{|c|c|c|c|c|c|c|c|}
\hline \multirow{2}{*}{$\begin{array}{l}\text { \# parallel } \\
\text { cases }^{b}\end{array}$} & \multirow{2}{*}{$\begin{array}{c}\% \text { cases with } \\
\text { same } \\
\text { outcome } \\
\end{array}$} & & \multicolumn{5}{|c|}{ Outcome in DE } \\
\hline & & & Infringed & $\begin{array}{l}\text { Not } \\
\text { infringed }\end{array}$ & Revoked & Settled & $\begin{array}{l}\text { Other } \\
\text { outcome }\end{array}$ \\
\hline \multicolumn{2}{|c|}{$\mathrm{DE}-\mathrm{UK}^{\ddagger}$} & $\begin{array}{c}\text { Outcome in } \\
\mathrm{UK}^{\ddagger}\end{array}$ & & & & & \\
\hline \multirow[t]{6}{*}{35} & 28.6 & Infringed & $0.0 \%$ & $0.0 \%$ & $66.7 \%$ & $0.0 \%$ & $0.0 \%$ \\
\hline & & Not infringed & $0.0 \%$ & $0.0 \%$ & $0.0 \%$ & $0.0 \%$ & $0.0 \%$ \\
\hline & & Revoked & $0.0 \%$ & $60.0 \%$ & $33.3 \%$ & $42.3 \%$ & $0.0 \%$ \\
\hline & & Settled & $100.0 \%$ & $40.0 \%$ & $0.0 \%$ & $34.6 \%$ & $0.0 \%$ \\
\hline & & $\begin{array}{l}\text { Other outcome } \\
\text { Total \# of }\end{array}$ & $0.0 \%$ & $0.0 \%$ & $0.0 \%$ & $23.1 \%$ & $0.0 \%$ \\
\hline & & cases & 1 & 5 & 3 & 26 & 0 \\
\hline \multicolumn{2}{|c|}{ DE-FR } & Outcome in FR & & & & & \\
\hline \multirow[t]{6}{*}{64} & 50.0 & Infringed & $0.0 \%$ & $0.0 \%$ & $0.0 \%$ & $2.4 \%$ & $0.0 \%$ \\
\hline & & Not infringed & $20.0 \%$ & $14.3 \%$ & $33.3 \%$ & $12.2 \%$ & $20.0 \%$ \\
\hline & & Revoked & $0.0 \%$ & $14.3 \%$ & $16.7 \%$ & $2.4 \%$ & $0.0 \%$ \\
\hline & & Settled & $80.0 \%$ & $71.4 \%$ & $16.7 \%$ & $73.2 \%$ & $80.0 \%$ \\
\hline & & $\begin{array}{l}\text { Other outcome } \\
\text { Total \# of }\end{array}$ & $0.0 \%$ & $0.0 \%$ & $33.3 \%$ & $9.8 \%$ & $0.0 \%$ \\
\hline & & cases & 5 & 7 & 6 & 41 & 5 \\
\hline \multicolumn{2}{|c|}{$\mathrm{DE}-\mathrm{NL}$} & $\begin{array}{l}\text { Outcome in } \\
\mathrm{NL}\end{array}$ & & & & & \\
\hline \multirow[t]{6}{*}{44} & 22.7 & Infringed & $50.0 \%$ & $0.0 \%$ & $20.0 \%$ & $50.0 \%$ & $0.0 \%$ \\
\hline & & Not infringed & $25.0 \%$ & $0.0 \%$ & $0.0 \%$ & $11.8 \%$ & $0.0 \%$ \\
\hline & & Revoked & $25.0 \%$ & $0.0 \%$ & $60.0 \%$ & $23.5 \%$ & $0.0 \%$ \\
\hline & & Settled & $0.0 \%$ & $100.0 \%$ & $20.0 \%$ & $14.7 \%$ & $0.0 \%$ \\
\hline & & $\begin{array}{l}\text { Other outcome } \\
\text { Total \# of }\end{array}$ & $0.0 \%$ & $0.0 \%$ & $0.0 \%$ & $0.0 \%$ & $0.0 \%$ \\
\hline & & cases & 4 & 1 & 5 & 34 & 0 \\
\hline
\end{tabular}

Columns for each jurisdiction-pair add up to $100 \%$; for example, of all parallel cases in DE-UK where infringement was found in Germany $(100 \%), 66.7 \%$ of these parallel cases in the UK held the patent infringed and $33.3 \%$ revoked the patent in the UK

a Parallel cases have been identified on the basis of patent numbers as well as claimants and defendants

b Number of cases for which information on case outcome available 